\title{
Puerarin Induces Hepatocellular Carcinoma Cell Apoptosis Modulated by MAPK Signaling Pathways in a Dose-dependent Manner
}

\author{
WEI-GUO ZHANG ${ }^{1}$, XIU-CAI YIN ${ }^{2}$, XIAO-FANG LIU ${ }^{1}$, KE-WEI MENG $^{1}$, \\ KUN TANG ${ }^{1}$, FEI-LONG HUANG ${ }^{1}$, GANG XU ${ }^{1}$ and $\mathrm{JIE} \mathrm{GAO}^{1}$ \\ ${ }^{1}$ Department of Hepatobiliary and Pancreatic Surgery, \\ Yantai Yuhuangding Hospital Affiliated to Qingdao University, Yantai, P.R. China; \\ ${ }^{2}$ Department of Hepatobiliary Surgery, People's Hospital of Rongcheng, Weihai, P.R. China
}

\begin{abstract}
Background/Aim: Puerarin possesses a battery of therapeutic values in diverse disorders, including proapoptotic actions in multiple cancers. Herein, we investigated the effects of puerarin on hepatocellular carcinoma (HCC) in vitro. Materials and Methods: MTT and flow cytometry were carried out to evaluate the viability and apoptosis of SMMC7721 HCC cells in the presence of different concentrations of puerarin. Moreover, expression levels, as well as phosphorylation status of several canonical components in mitogen-activated protein kinase (MAPK) pathways, including extracellular signal-regulated kinase $1 / 2$ (ERK1/2), c- Jun Nterminal kinase $(J N K)$, p38, were measured by reverse transcription and quantitative real-time polymerase chain reaction (RT-PCR) and western blot analysis at indicated time intervals. Results: Puerarin inhibited proliferation of SMMC7721 cells and promoted their apoptosis in a dose-and timedependent fashion $(p<0.05)$. Both the expression and phosphorylation levels of MAPK proteins were dramatically increased on puerarin treatment. Conclusion: Puerarin could be employed as a potential anti-carcinogen that exhibits proapoptotic effects on HCC cells, in a dose-and time-dependent manner, with emphasis on MAPK pathways whose initiation may contribute to this process.
\end{abstract}

Hepatocellular carcinoma (HCC) is the sixth most frequent cancer worldwide and has nowadays become more prevalent

Correspondence to: Wei-guo Zhang, MD, Department of Hepatobiliary and Pancreatic Surgery, Yantai Yuhuangding Hospital Affiliated to Qingdao University, 20 East Yuhuangding Road, Zhifu, Yantai, Shandong 264000, P.R. China. Tel: +86 05356691999, Fax: +8605356240341, e-mail: zhangwg1970@163.com

Key Words: Puerarin, hepatocellular carcinoma (HCC), apoptosis, MAPK pathway. in developing countries (1) with high incidence and mortality rates $(2,3)$. Conventional chemotherapeutic regimens occupy a principal role in advanced or metastatic tumors, in particular for reasons of organ preservation. However, multidrug resistance (MDR), whether intrinsic or acquired, to structurally-diverse and mechanistically-unrelated antitumor drugs is deemed as a major obstacle in the chemotherapeutic management of $\operatorname{HCC}(4,5)$. Besides this, severe cytotoxic effects caused by the systemic combined chemotherapy also limit its broad application. Therefore, it is urgently desired to develop novel and effective drugs for patients with HCC.

Puerarin, 7-hydroxyl-3-(4-hydroxyphenyl)-1- benzopyran4- one 8- $\beta$-D-glucopyranoside $\left(\mathrm{C}_{12} \mathrm{H}_{20} \mathrm{C}_{9}\right)$, is a major isoflavonoid compound extracted from the root of a wild leguminous creeper exerting a wide array of beneficial biological functions, such as neuroprotection (6), antiosteoporotic (7), anti-oxidant (8), anti-viral (9, 10), antiinflammatory (11) and antitumor $(12,13)$ activities. Antitumor and anti-HIV activities of flavonoids and their analogues were initially discovered by Wang and coworkers in 1998 (10), which provided a promising and continuing source of potential antitumor agents. Since then, an immense number of studies, focusing on chemical features of puerarin with antitumor activity, have been launched. As Zeng et al. reported, the combination of 5-fluorouracil (5-FU) with puerarin in advanced-stage HCC could enhance the efficacy and decrease the toxicity of 5-FU, a chemotherapeutic compound frequently used in cancer treatment (14). In addition, the in vivo anticancer activity of the puerarin nanosuspensions was observed in HT-29 cancer-bearing mice (15) and corroborated in vitro in HT-29 cells. Other researchers also found that Pueraria radix metabolites inhibited the growth of breast cancer cells with high efficacy (16). On the other hand, puerarin could suppress the expression of multidrug resistance 1 (MDR1), a glycoprotein 
involved in pleiotropic drug resistance (17). Therefore, its use could aid patients in alleviating resistance to diverse chemotherapeutic drugs whilst improving the final outcome of treatment in clinical practice.

Although these data provide compelling evidence that puerarin possesses anticancer activity and significant antiproliferative and apoptotic effects, the detailed molecular mechanisms remain to be unraveled. In the present study, we evaluated the apoptosis-promoting effect of puerarin on human SMMC7721 hepatocellular carcinoma cells, providing new insights into the mechanisms involved, at least in part, underlying its antitumor activity against refractory $\mathrm{HCC}$.

\section{Materials and Methods}

Cell culture. Human- derived SMMC-7721 HCC cell line was purchased from the Cell Bank of Chinese Academy of Sciences (Shanghai, PR China). The cells were maintained in RPMI 1640 medium (Gibco, Carlsbad, CA, USA) supplemented with 10\% fetal bovine serum (FBS; Gibco) and antibiotics (100 $\mathrm{mg} / \mathrm{ml}$ of ampicillin and $100 \mathrm{mg} / \mathrm{ml}$ of streptomycin; Beyotime Institute of Biotechnology, Shanghai, China). Cells were grown in a humidified atmosphere of $5 \% \mathrm{CO}_{2}$ at $37^{\circ} \mathrm{C}$, with the medium being changed after $48 \mathrm{~h}$ to remove non-adherent cells

MTT assay. Cell viability was tested by performing MTT colorimetric assay. Briefly, cells in monolayer culture were plated at $5 \times 10^{3}$ cells/well into a 96-well microliter plate and treated with escalating concentrations of puerarin (Sigma-Aldrich, St. Louis, MO, USA) stepwise $(0,50,100,250,500,1,000,1,500,2,000 \mu \mathrm{g} / \mathrm{ml})$ for various time intervals $(12,24,48 \mathrm{~h})$. Fifty microliters of MTT solution was added and the culture was incubated for additional $4 \mathrm{~h}$ at $37^{\circ} \mathrm{C}$. After aspiration of MTT solution off the culture and addition of $200 \mathrm{ml}$ DMSO for solubilization, the absorbance was measured at $492 \mathrm{~nm}$ using a microplate reader (Multiskan MK3; Thermo Labsystems, Philadelphia, PA, USA). All assays were performed in quintuplicate and repeated at least three times.

Apoptotic rate determination by flow cytometry. The rates of apoptotic cells were evaluated by flow cytometry analysis. Briefly, cells were treated with $0,500,1,000$ or $1,500 \mu \mathrm{g} / \mathrm{ml}$ of puerarin for either 12 or $24 \mathrm{~h}$. The cells were stained with an Annexin Vfluorescein isothiocyanate apoptosis detection kit (KeyGEN Biotech, Nanjing, China) and subsequently subjected to flow cytometry analysis (Becton-Dickinson, Mountain View, CA, USA).

Morphological change analysis. Morphological changes of cells were observed under a Leica DM 500B fluorescence microscope (Leica Microsystems Inc., Buffalo Grove, IL, USA) in the presence of indicated concentrations of puerarin $(0,500,1,000$ or $1,500 \mu \mathrm{g} / \mathrm{ml})$ for 12 or $24 \mathrm{~h}$.

Mitochondrial membrane potential (MMP). Following $24 \mathrm{~h}$ exposure to various concentrations of puerarin $(0,500,1,000$ and $1,500 \mu \mathrm{g} / \mathrm{ml})$, the cells were subjected to flow cytometry analysis using a BD FACScan instrument coupled with Rhodamine 123 dye to detect MMP alterations.
Detection of reactive oxygen species (ROS). ROS fluorescence intensity was determined in cells treated with indicated concentrations of puerarin $(0,500,1,000$ and $1,500 \mu \mathrm{g} / \mathrm{ml})$ for $12 \mathrm{~h}$ by performing flow cytometry analysis with excitation and emission wavelengths at $490 \mathrm{~nm}$ and $520 \mathrm{~nm}$, respectively.

Reverse transcription and quantitative real-time polymerase chain reaction $(P C R)$. Total RNA was isolated from puerarin- treated cells by using Trizol reagent (Invitrogen, Carlsbad, CA, USA), according to the manufacturer's recommended protocol. Using $2 \mu \mathrm{g}$ RNA as template, reverse transcription was carried out with Rever-Tra-Ace$\alpha$-Transcriptase (Toyobo, Tokyo, Japan) to synthesize the complementary DNA (cDNA). To evaluate apoptosis-associated gene expression, quantities of target genes were normalized to that of the housekeeping gene GAPDH, which was used as internal control. The sequences of primers used in this study are listed below:

\section{Caspase-3, 5'-AACTGGACTGTGGCATTGAG-3' \\ 5'-ACAAAGCGACTGGATGAACC-3'; \\ Caspase-8, 5'-CTGGGAGAAGGAAAGTTG-3' \\ 5'-TTGGAGAGTCCGAGATTG-3'; \\ Caspase-9, 5'-GGAAGAGGGACAGATGAATG-3' \\ 5'-TTGTTTGGCACCACTCAG-3'; \\ AIF, 5'-GCTACAAGCACGCTCTAACATC-3' \\ 5'-CAGCCAATCTTCCACTCACAAC-3'; GAPDH, 5'-CACCCACTCCTCCACCTTTG-3' 5'-CCACCACCCTGTTGCTGTAG-3'.}

Quantitative PCR was carried out using an ABI 7300 Thermocycler using the SYBR ${ }^{\circledR}$ Premix Ex Taq ${ }^{\text {TM }}$ II (Tli RNaseH Plus) (Takara, Tokyo, Japan). The amplification profile was one cycle at $95^{\circ} \mathrm{C}$ for $10 \mathrm{~min}$, followed by 40 cycles of denaturation $95^{\circ} \mathrm{C}$ for $15 \mathrm{~s}$, hybridization annealing/extension at $60^{\circ} \mathrm{C}$ for $45 \mathrm{~s}$. PCR products were visualized on $1.0 \%(\mathrm{w} / \mathrm{v})$ agarose gels stained with ethidium bromide. The mRNA levels were determined by measuring absorbance value using 'Quantity one 4.6' software (BioRad Laboratories, Inc., Hercules, CA, USA). The comparative $\mathrm{Ct}(2-\Delta \Delta \mathrm{Ct})$ method was introduced to calculate the relative-fold changes of mRNA expression. All reactions were performed in triplicate and values reported are means \pm standard deviations (SDs).

Protein extraction and western blotting analysis. Cultures were washed three times with PBS and sequentially harvested cells were pelleted and re-suspended in radioimmunoprecipitation assay (RIPA) lysis buffer containing 1\% phenylmethylsulfonyl fluoride (PMSF) protease inhibitor, followed by incubating on ice for 1 hour. Lysates were subjected to ultrasonication on ice for further lysing and cell debris was removed by centrifugation at $12,000 \mathrm{rpm}$ for 10 min at $4^{\circ} \mathrm{C}$. After centrifugation, protein supernatant was kept at $-80^{\circ} \mathrm{C}$ for future analysis.

For immunoblotting, $20 \mu \mathrm{g}$ protein samples were separated on $10 \%$ sodium dodecyl sulfate-polyacrylamide gel electrophoresis (SDS-PAGE) and transferred to polyvinylidene difluoride (PVDF) membranes at $60 \mathrm{~V}$ for $1 \mathrm{~h}$ at $4^{\circ} \mathrm{C}$. The membranes were blocked with $5 \%$ non-fat milk and then incubated overnight at $4{ }^{\circ} \mathrm{C}$ with mouse monoclonal primary antibodies against human AKT (1:1000; Cell Signaling Technology, Inc., Danvers, MA, USA), phosphorylated AKT (p-AKT; Santa Cruz Biotechnology, Inc., Santa Cruz, CA, USA), P38 (1:1,000; Abcam, Cambridge, CA, USA), p-P38 (1:1,000; Cell Signaling Technology, Inc.), 
extracellular signal-regulated kinase 1 (ERK1, 1:1000; Abcam), pERK1 (1:1,000; Cell Signaling Technology, Inc.), c- Jun N- terminal kinase (JNK; 1:1,000; Abcam), p-JNK, Caspase-3 (1:800; Abcam) or AIF (1:2,000; Fermentas Inc., Burlington, ON, Canada), Caspase8 (1:1,000; Abcam), Caspase-9 (1:1,000; Abcam), GAPDH (Santa Cruz Biotechnology, Inc.), followed by rinsing 3 times with phosphate-buffered saline with Tween 20 (PBST0 for $15 \mathrm{~min}$ and subsequently incubated with horseradish peroxidase (HRP)conjugated secondary bovine anti-mouse $\operatorname{IgG}(1: 2,000$; Biosynthesis Biotech Co., Ltd. Beijing, China) at room temperature for 1 hour before washing and before visualization using enhanced chemiluminescence (ECL) reagents (Millipore, Billerica, MA, USA). LabWorks Image Acquisition and Analysis Software version 4.5 (Bio-Medicine, http://www.Bio-medicine.org) was used to quantify band intensities.

Statistical analysis. All data are presented as means \pm SDs. Significance between various treatment samples was calculated by Student's $t$-test for two group comparisons or one-way analysis of variance when more than two groups were compared. All tests performed were two-sided. Differences between the mean values were evaluated with a minimal significance of $p$-values $<0.05$. All statistical analyses were carried out using the SPSS 16.0 software (SPSS, Inc., Chicago, IL, USA).

\section{Results}

Puerarin inhibited SMMC-7721 cell viability in a dose- and time-dependent way. To investigate the biological effects of puerarin on tumor cell viability, we first performed an MTT assay in the presence of escalating concentrations of puerarin stepwise for various time intervals $(12,24,48 \mathrm{~h}): 50,100$, $250,500,1,000,1,500,2,000 \mu \mathrm{g} / \mathrm{ml}$. As shown in Figure 1A, the growth rate of SMMC-7721 cells was retarded upon treatment with puerarin, indicative of the inhibitory function of peurarin in hepatocellular carcinoma proliferation. In particular, as puerarin concentration or time interval increased, cell proliferation rate declined more sharply and, when the concentration reached more than $500 \mu \mathrm{g} / \mathrm{ml}$, the proliferation of SMMC-7721 cells dropped dramatically. We, thus, concluded that puerarin negatively regulated the viability of hepatocellular carcinoma SMMC-7721 cells in a time- and dose-dependent fashion.

SMMC-7721 cell apoptosis rate was prompted upon puerarin treatment. We next contemplated that the retarded proliferation rate might be contributed by cell apoptosis. The assumption was validated further by Annexin V/PI analysis (Figure 1B) where FACS measurement indicated a marked increase in cellular apoptosis detected in puerarin-treated SMMC-7721 cells as compared with control cells. In agreement with our findings observed in cell viability assay, high concentration of puerarin brought about dramatically increased apoptotic rates in SMMC-7721 in a dose- and time-dependent fashion. In addition, exposure of SMMC7721 to puerarin treatment led to marked morphologic changes that were comparable to untreated cells. These changes were histologically characterized by apoptotic body formation and condensation of nuclear chromatin, at least in part, as visualized by staining with an apoptosis-specific dye, Hoechst 33258 (Figure 1C). These, in aggregate, indicated that the negatively inhibitory effect of high concentration of puerarin on SMMC-7721 cell viability was mainly caused by induction of cell apoptosis.

Puerarin induced mitochondria-mediated apoptosis in a dose- and time-dependent manner. The breakdown of MMP occurs at an early stage of the apoptotic process and precedes mitochondrial membrane integration disruption, which ultimately resulted in MMP loss. Flow cytometry analysis coupled with Rho-123 staining was utilized as a tool towards evaluating MMP alterations in SMMC-7721 cells, following $24 \mathrm{~h}$ of treatment with various concentrations of puerarin $(0$, $500,1,000$ and $1,500 \mu \mathrm{g} / \mathrm{ml})$. As expected, with a gradual increase in puerarin concentration, a significant depolarization of MMP in SMMC-7721 cells was observed (Figure 1D), resulting in an increase in the permeability of the mitochondrial membrane from which pro-apoptotic molecules were released.

Theoretically, mitochondrial dysfunction is preceded by excessive reactive oxygen species (ROS) production and consequent oxidative stress since they are mainly generated in the mitochondria. As depicted in Figure 1E, upon puerarin treatment for $12 \mathrm{~h}$, the intracellular accumulation of ROS aggrandized substantially, indicative of mitochondriamediated apoptosis in SMMC-7721 cells induced by 500 , 1,000 and $1,500 \mu \mathrm{g} / \mathrm{ml}$ of puerarin.

Mitogen-activated protein kinase (MAPK) pathways were activated during puerarin suppressing SMMC-7721 apoptosis. We expected to unveil the hitherto unreported mechanistic underpinnings of how puerarin negatively affected cell apoptosis in hepatocellular carcinoma. To this end, we first tested several apoptosis-associated hallmarks, including caspase-3, -8, -9, as well as $A I F$, in puerarintreated SMMC-7721 cells by quantitative RT-PCR. Histogram in Figure 1D allows delineation of a significant increase in the expression level of all aforementioned genes in the presence of 30 (inhibitory concentration (IC) ${ }_{25}$ ), 500 $\left(\mathrm{IC}_{50}\right)$ and 2,000 $\left(\mathrm{IC}_{75}\right) \mu \mathrm{g} / \mathrm{ml}$ puerarin in a dose- dependent manner. In particular, the levels of caspase- 9 mRNA expression increased by approximately 25 - and 50-fold after a 12-h exposure to 500 and $2,000 \mu \mathrm{g} / \mathrm{ml}$ of puerarin, respectively (Figure 2A). Subsequently, the RT-PCR results were further corroborated by immunoblotting analysis, which was also employed to detect the phosphorylation status of key kinases involved in three predominant subfamilies of MAPK signaling pathways. SMMC-7721 cells were treated with $500 \mu \mathrm{g} / \mathrm{ml}$ of puerarin for 1,3 or $6 \mathrm{~h}$, prior to western 
A

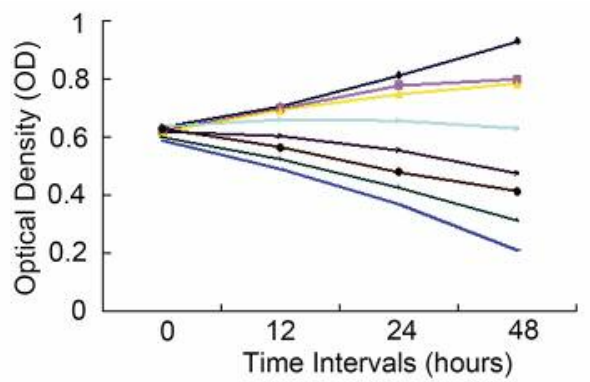

$+0 \mu \mathrm{g} / \mathrm{ml}$
$-50 \mu \mathrm{g} / \mathrm{ml}$
$-100 \mu \mathrm{g} / \mathrm{ml}$
$-250 \mu \mathrm{g} / \mathrm{ml}$
$-500 \mu \mathrm{g} / \mathrm{ml}$
$-1000 \mu \mathrm{g} / \mathrm{ml}$
$-1500 \mu \mathrm{g} / \mathrm{ml}$
$-2000 \mu \mathrm{g} / \mathrm{ml}$

B
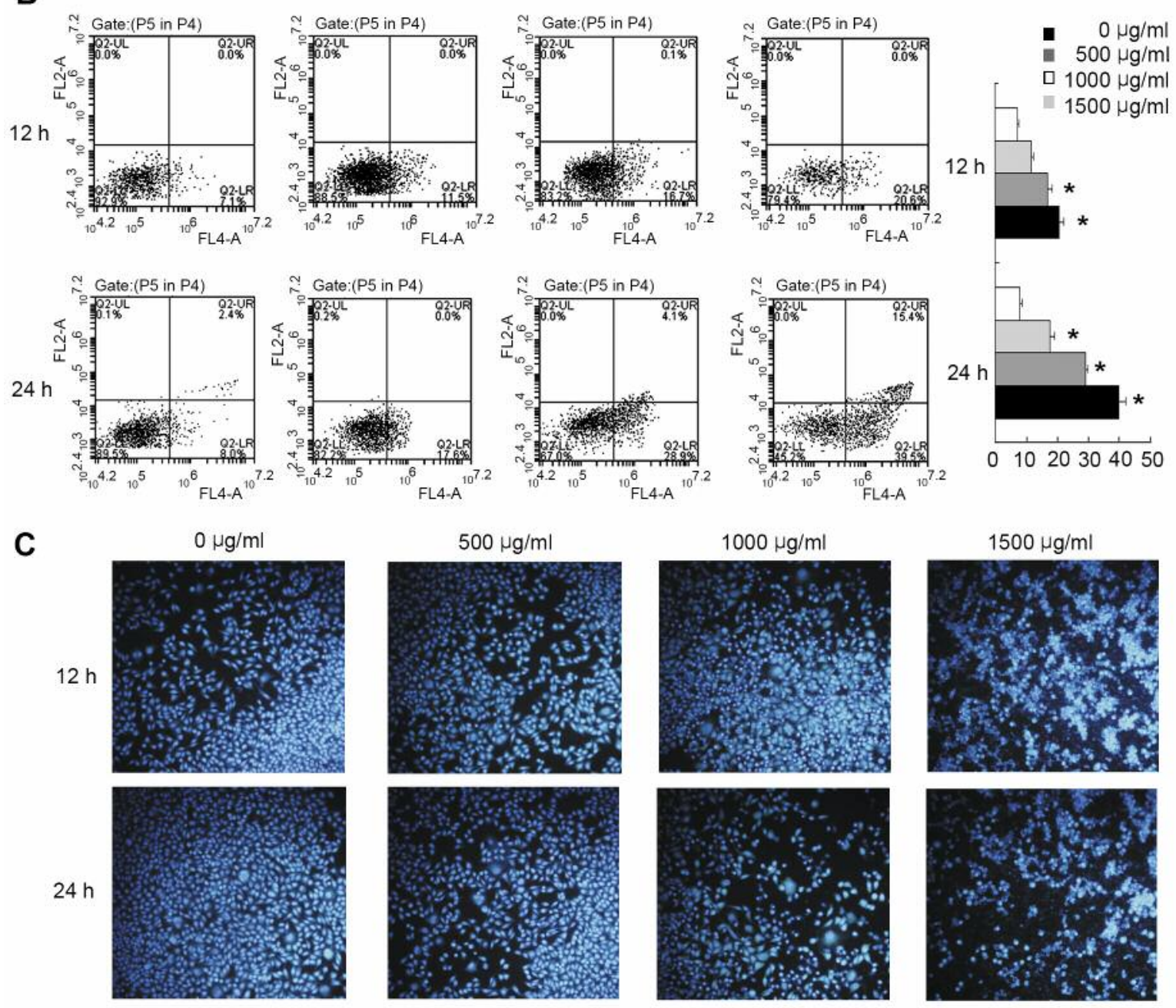

D

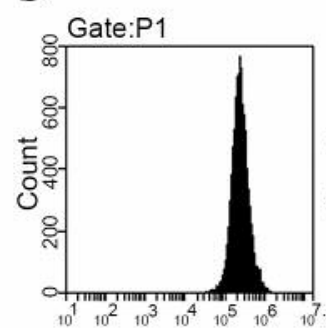

FL2-A

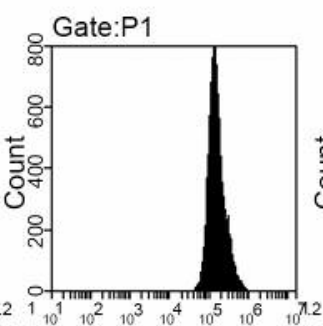

FL2-A

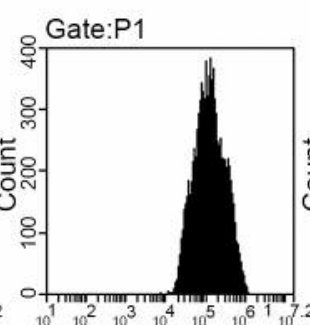

FL2-A

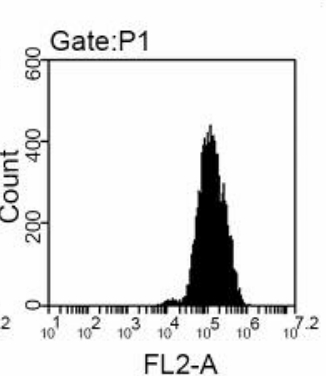

FL2-A

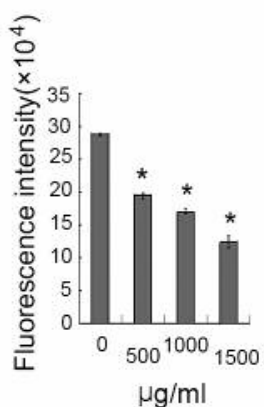

Figure 1. Continued 
E

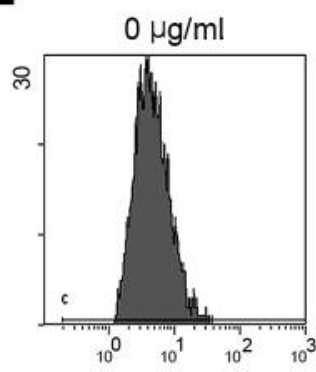

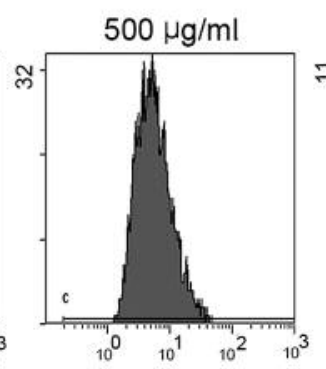
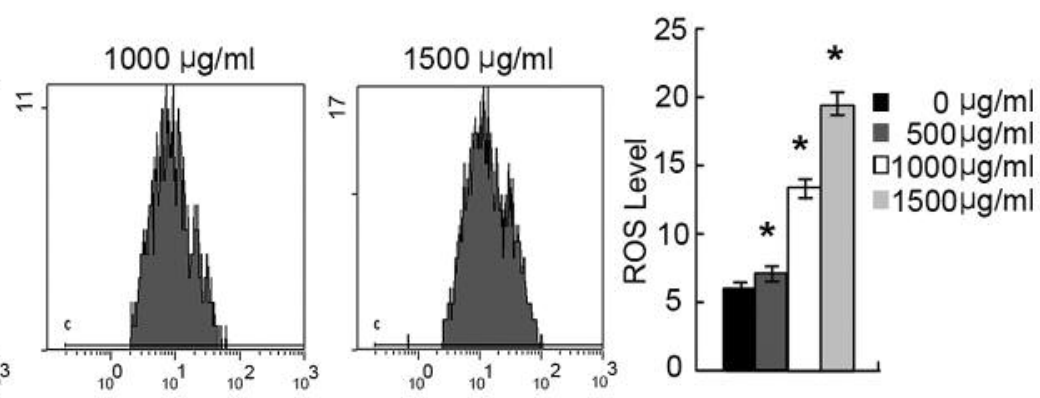

Figure 1. Puerarin inhibited SMMC-7721 cell viability and promoted apoptosis in a dose-and time-dependent way. (A) MTT assays revealed the retarded SMMC-7721 proliferation rate caused by puerarin. Viability of SMMC-7721 cells treated with various concentrations of puerarin was measured at indicated time intervals. Data are presented as mean values. (B) Representative plots for the flow cytometry analysis of SMMC-7721 cells incubated for 12 or $24 \mathrm{~h}$ with 0,500, 1,000 and 1,500 $\mathrm{\mu g} / \mathrm{ml}$ puerarin. All experiments were conducted in triplicate and data are presented as means \pm SDs. ${ }^{*} p<0.05$, compared to untreated cells. (C) Morphological changes of Hoechst 33258-stained SMMC-7721 cells exposed to puerarin $(0,500,1,000$ and 1,500 $\mathrm{g} / \mathrm{ml})$ for 12 or $24 \mathrm{~h}$ were visualized by fluorescence microscopy. Magnification $\times 200$. Mitochondria-dependent apoptosis occurrence of SMMC-7721 cells was increased when exposed to various concentrations $(0,500,1,000$ and 1,500 $\mu \mathrm{g} / \mathrm{ml})$ of puerarin for $24 \mathrm{~h}$, as assessed by $(D)$ mitochondrial membrane potential $(M M P)$ level and $(E)$ reactive oxygen species $(R O S)$ production. ${ }^{*} p<0.05$.

blot analysis. Similar to mRNA expression, protein products encoded by the apoptosis-associated genes were aggrandized on puerarin's stimulation in a time-dependent manner. Moreover, a prolonged duration of puerarin treatment was accompanied by occurrence of ERK, JNK and p38 phosphorylation events (Figure 2B). Additionally, the phosphorylation level of AKT, a predominant regulator that participates in transcriptional regulation of the anti-apoptotic protein B-cell lymphoma 2, was enhanced in the presence of puerarin. Activation of the phosphoinositide 3kinase/AKT signaling pathway was reported to take part in puerarin protecting against iodide-induced SH-SY5Y neuroblastoma cell death (18). AKT phosphorylation, in our study, ought to be an adaptive response to puerarin promoting SMMC-7721 cell apoptosis. These results from our in vitro study support a concentration- and timedependent relationship between puerarin exposure, as well as MAPK-mediated cell apoptosis initiation.

\section{Discussion}

Pueraria, a bioactive isoflavone-C-glucoside deriving from the root of Pueraria lobata (Willd.) Ohwi, has been widely employed as an traditional oriental medicinal herb in treating cardiovascular disorders, alcohol-induced liver injury and cirrhosis, diabetes, Parkinson's disease, Alzheimer's disease, osteonecrosis and endometriosis (19), due to its versatile pharmacological properties, including cardioprotection, neuroprotection, antioxidant, antiinflammation, attenuating insulin resistance, etc. Besides these biological activities, its potential but unilluminated anticancer capacity has attracted the attention of a myriad of researchers throughout the world.

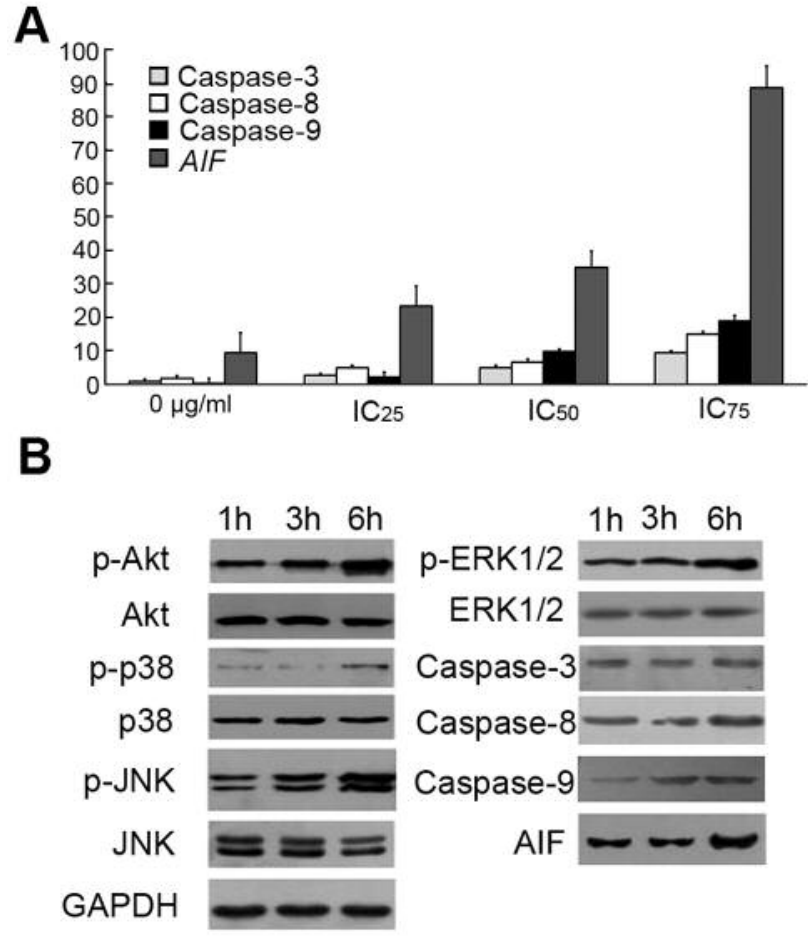

Figure 2. Expression of apoptosis-associated genes and phosphorylation of MAPKs were obviously elevated upon puerarin treatment. (A) SMMC-7721 cells treated with $30\left(I_{25}\right), 500\left(I C_{50}\right)$, and 2,000 $\left(I C_{75}\right) \mu \mathrm{g} / \mathrm{ml}$ of puerarin for $12 \mathrm{~h}$ were subjected to quantitative realtime polymerase chain reaction ( $q R T-P C R)$. GAPDH was used as an internal control and error bars represent the standard deviation from triplicates. (B) Immunoblot assay performed at indicated time intervals to evaluate protein expression levels in SMMC-7721 cells in the presence of $500 \mu \mathrm{g} / \mathrm{ml}$ puerarin. $\mathrm{IC}_{50}$, half-maximal inhibitory concentration (causing $50 \%$ reduction); $I C_{25}$, inhibitory concentration causing $25 \%$ reduction; $I C_{75}$, inhibitory concentration causing $75 \%$ reduction. 
Puerarin exhibits an estrogen-like structure and moderates estrogenic activity whist acting as a phytoestrogen to facilitate osteoblast proliferation and differentiation (20), as well as suppress osteoblastic apoptosis (21). On the other hand, puerarin also exerts bone-preventing action independent of estrogen receptor-mediated pathways (22). Puerarin at $0.12 \mathrm{mg} / \mathrm{kg}$ is able to protect dopaminergic neurons against cell death via repression of apoptosis and enhancement of neurotrophic factor expression in rats with Parkinson's disease (23). Pre-treatment with peurarin attenuates cell apoptosis in vitro, while its administration can reverse a series of pathological processes, including an increase in ROS formation, cell apoptosis and, particularly, activation of p38 and JNK in sporadic Alzheimer's disease (24).

MAPK pathways are evolutionarily conserved kinase modules that orchestrate cellular responses to extra stimuli and mediate fundamental cellular processes, including cell growth, proliferation, apoptosis, differentiation and migration. Canonical MAPK pathways are generally constituted of at least three subfamilies of MAPKs that have been wellcharacterized, namely, extracellular signal-regulated kinase (ERK), c-Jun N-terminal kinase (JNK) and p38/MAPK. All these MAPK signaling pathways are involved in apoptosis regulation and aberrations in these pathways can cause apoptosis evasion $(25,26)$.

In this study, we found that puerarin reduced the viability of human-derived hepatocellular carcinoma SMMC-7721 cells in a dose- and time-dependent manner and that puerarin-induced cytotoxicity mainly resulted from induction of cell apoptosis. Remarkably, the phosphorylation levels of ERK1, JNK and p38 were dramatically elevated after exposure to $500 \mu \mathrm{g} / \mathrm{ml}$ of puerarin for $6 \mathrm{~h}$. This indicated that, concerning apoptosis, puerarin is related with ERK1, JNK and p38 activation and we, thus, assumed that these MAPK pathways were responsible for mitochondriamediated apoptosis in SMMC-7721 cells.

Similar results were also reported by Liu et al. in BxPC human pancreatic cancer cells and type II pulmonary epithelial A549 cells, where ERK, JNK and p38 MAPK pathways were activated by oleanolic acid (OA), a nutritional component in vegetarian food and medicinal herbs (27), whereas blockage of p38 MAPK pathway rescued the proapoptotic effect of OA on corresponding cells. In human hepatoblastoma cancer HepG2 cells, a flavonoid compound, isoorientin, could stimulate the phosphorylation of JNK and p38 (28), consistent with our results. These data, in aggregate, suggested that puerarin might promote SMMC-7721 cell apoptosis through activating multiple MAPK pathways.

Notably, in a previous study, puerarin failed to stimulate apoptosis in other types of normal cells. For instance, treatment with a low dose $(25 \mu \mathrm{mol} / \mathrm{l})$ of puerarin alleviated apoptosis of rat hippocampal neurons induced by highglucose, which specifically simulated the internal environment of diabetic patients that usually lead to cognitive dysfunction, with the p-p38/p38 and $\mathrm{p}-\mathrm{JNK} / \mathrm{JNK}$ ratios being downregulated in the puerarin-treated group (21). Puerarin also acted through ERK signaling cascades to protect human osteoblasts from serum-free-induced apoptosis and displayed maximal anti-apoptotic effect at a concentration of $0.01 \mu \mathrm{mol} / 1$ (21). We, therefore, assumed that puerarin exerts different effects via MAPK signaling pathways dependent on drug concentration.

More intriguingly, increasing published evidence demonstrates the apparent crosstalk of MAPK signaling with other signal transduction pathways in tumorigenesis, chondrogenesis, as well as other physiological and pathological processes, thus implicating proliferation, differentiation and apoptosis (29-31). Consequently, we reasonably assumed that puerarin exhibits dual biological effects on cell apoptosis via MAPK pathways by interacting with additional divergent pathways. To this end, we will attempt to investigate proteins' interactions with AMPactivated protein kinases (AMPKs), including Akt, p38 and JNK, in the future.

In summary, the present study revealed that high concentrations of puerarin suppressed proliferation and promoted apoptosis in SMMC-7721 cells via MAPK scaffold, interacting with molecules that were involved in other crucial signaling pathways, in a time- and dosedependent manner.

\section{Conflicts of Interest}

The Authors confirm that this article's content has no conflicts of interest.

\section{References}

1 Llovet JM, Burroughs A and Bruix J: Hepatocellular carcinoma. Lancet 362: 1907-1917, 2013.

2 Roberts LR: Sorafenib in liver cancer - Just the beginning. N Engl J Med 359: 420-422, 2008.

3 El-Serag HB and Rudolph KL: Hepatocellular carcinoma: Epidemiology and molecular carcinogenesis. Gastroenterology 132: 2557-2576, 2007.

4 Ryu SH and Chung YH: How to overcome multidrug resistance in chemotherapy for advanced hepatocellular carcinoma. Liver Int 30: 496-498, 2010.

$5 \mathrm{Gu}$ W, Fang FF, Li B, Cheng BB and Ling CQ: Characterization and resistance mechanisms of a 5-fluorouracil- resistant hepatocellular carcinoma cell line. Asian Pac J Cancer Prev 13(9): 4807-4814, 2012.

6 Tian F, Xu LH, Zhao W, Tian LJ and Ji XL: The neuroprotective mechanism of puerarin treatment of acute spinal cord injury in rats. Neurosci Lett 543: 64-68, 2013.

7 Michihara S, Tanaka T, Uzawa Y, Moriyama T and Kawamura Y: Puerarin exerted anti-osteoporotic action independent of estrogen receptor-mediated pathway. J Nutr Sci Vitaminol Tokyo 58(3): 202-209, 2012. 
8 Wolfe $\mathrm{K}, \mathrm{Wu} \mathrm{X}$ and Liu RH: Antioxidant activity of apple peels J Agric Food Chem 51: 609-614, 2003.

9 Arao T, Udayama M, Kinjo J, Nohara T, Funakoshi T and Kojima S: Preventive effects of saponins from Puerariae radix (the root of Pueraria lobata Ohwi) on in vitro immunological injury of rat primary hepatocyte cultures. Biol Pharm Bull 20: 988-991, 1997.

10 Wang HK, Xia Y, Yang ZY, Natschke SL and Lee KH: Recent advances in the discovery and development of flavonoids and their analogues as antitumor and anti-HIV agents. Adv Exp Med Biol 439: 191-225, 1998.

11 Theoharides TC, Alexandrakis M, Kempuraj D and Lytinas M: Anti-inflammatory actions of flavonoids and structural requirements for new design. Int J Immunopathol Pharmacol 14: 119-127, 2001.

12 Joshi KS, Rathos MJ, Joshi RD, Sivakumar M, Mascarenhas M, Kamble S, Lal B and Sharma S: In vitro antitumor properties of a novel cyclin-dependent kinase inhibitor, P276-00. Mol Cancer Ther 6: 918-925, 2007.

$13 \mathrm{Yu} \mathrm{Z}$ and $\mathrm{Li} \mathrm{W}$ : Induction of apoptosis by peurarin in colon cancer HT-29 cells. Cancer Lett 238: 53-60, 2006.

14 Zeng YP, Yang ZR, Guo XF, Jun W and Dong WG: Synergistic effect of puerarin and 5-fluorouracil on hepatocellular carcinoma. Oncol Lett 8(6): 2436-2442, 2014.

15 Wang Y, Ma Y, Zheng Y, Song J, Yang X, Bi C, Zhang D and Zhang Q: In vitro and in vivo anticancer activity of a novel puerarin nanosuspension against colon cancer, with high efficacy and low toxicity. Int J Pharm 441(1-2): 728-735, 2013.

16 Lin YJ, Hou YC, Lin CH, Hsu YA, Sheu JJ, Lai CH, Chen BH, Lee Chao PD, Wan L and Tsai FJ: Puerariae radix isoflavones and their metabolites inhibit growth and induce apoptosis in breast cancer cells. Biochem Biophys Res Commun 378(4): 683688,2009

17 Hien TT, Kim HG, Han EH, Kang KW and Jeong HG: Molecular mechanism of suppression of MDR1 by puerarin from Pueraria lobata via NF-kappaB pathway and cAMP-responsive element transcriptional activity-dependent up-regulation of AMP-activated protein kinase in breast cancer MCF-7/adr cells Mol Nutr Food Res 54(7): 918-928, 2010.

18 Zhu G, Wang X, Wu S and Li Q: Involvement of activation of $\mathrm{PI} 3 \mathrm{~K} / \mathrm{Akt}$ pathway in the protective effects of puerarin against $\mathrm{MPP}^{+}$-induced human neuroblastoma SH-SY5Y cell death. Neurochem Int 60: 400-408, 2012.

19 Zhou YX, Zhang H, Peng C. Puerarin: A Review of pharmacological effects. Phytother Res 28(7): 961-975, 2014.

20 Wang PP, Zhu XF, Yang L, Liang H, Feng SW and Zhang RH: Puerarin stimulates osteoblasts differentiation and bone formation through estrogen receptor, p38 MAPK, and Wnt/ $\beta$ catenin pathways. J Asian Nat Prod Res 14(9): 897-905, 2012.
21 Liu LJ, Liu LQ, Bo T, Li SJ, Zhu Z, Cui RR and Mao DA: Puerarin suppress apoptosis of human osteoblasts via ERK signaling pathway. Int J Endocrinol 2013: 786574, 2013.

22 Zhu G, Wang X, Chen Y, Yang S, Cheng H, Wang N and Li Q: Puerarin protects dopaminergic neurons against 6hydroxydopamine neurotoxicity via inhibiting apoptosis and upregulating glial cell line-derived neurotrophic factor in a rat model of Parkinson's disease. Planta Med 76: 1820-1826, 2010.

23 Zhang H, Liu Y, Lao M, Ma Z and Yi X: Puerarin protects Alzheimer's disease neuronal cybrids from oxidant-stress induced apoptosis by inhibiting pro-death signaling pathways. Exp Gerontol 46: 30-37, 2011.

24 Wada T and Penninger JM: Mitogen-activated protein kinases in apoptosis regulation. Oncogene 23(16): 2838-2849, 2004.

25 Dangle PP, Zaharieva B, Jia H and Pohar KS: Ras-mapk pathway as a therapeutic target in cancer - Emphasis on bladder cancer. Recent Pat Anticancer Drug Discov 4: 125-136, 2009.

26 Liu J, Wu N, Ma LN, Zhong JT, Liu G, Zheng LH and Lin XK: p38 MAPK signaling mediates mitochondrial apoptosis in cancer cells induced by oleanolic acid. Asian Pac J Cancer Prev 15(11): 4519-4525, 2014.

27 Li Yuan, Jing Wang, Haifang Xiao, Wanqiang Wu, Yutang Wang and Xuebo Liu: MAPK signaling pathways regulate mitochondrial-mediated apoptosis induced by isoorientin in human hepatoblastoma cancer cells. Food Chem Toxicol 53: 6268, 2013.

$28 \mathrm{Xu} \mathrm{X}$, Wang J, Zhang H, Tian G and Liu Y: Puerarin reduces apoptosis in rat hippocampal neurons culture in high glucose medium by modulating the $\mathrm{p} 38$ mitogen activated protein kinase and c-Jun N-terminal kinase signaling pathways. J Tradit Chin Med 36(1): 78-84, 2016.

29 Guardavaccaro D and Clevers H: Wnt/beta-catenin and mapk signaling: Allies and enemies in different battlefields. Sci Signal 5(219): pe15, 2012.

30 Zhang Y, Pizzute T and Pei M: A review of crosstalk between mapk and wnt signals and its impact on cartilage regeneration. Cell Tissue Res 358: 633-649, 2014.

31 Wang L, Li J, Zhao H, Hu J, Ping Y, Li F, Lan Y, Xu C, Xiao Y and Li X: Identifying the crosstalk of dysfunctional pathways mediated by lncRNAs in breast cancer subtypes. Mol Biosyst 12(3): 711-720, 2016.
Received May 19, 2017

Revised June 9, 2017

Accepted June 12, 2017 